\title{
DESENVOLVIMENTO DO PROCEDIMENTO ÓTIMO DA PARTIDA DE UM SISTEMA DE COLUNAS DE DESTILAÇÃO TERMICAMENTE ACOPLADAS
}

\author{
A. L. U. VASCONCELOS ${ }^{1}$, I. C. NUNES $^{1}$, L. G. S. VASCONCELOS ${ }^{1}$ e R. P. BRITO ${ }^{1}$ \\ ${ }^{1}$ Universidade Federal de Campina Grande, Unidade Acadêmica de Engenharia Química \\ E-mail para contato: angelalucinia@yahoo.com.br
}

\begin{abstract}
RESUMO - As operações de partida de processos químicos e em particular, colunas de destilação, representam interessantes problemas de operabilidade, controle e simulação. Durante a operação de partida de uma coluna de destilação ocorrem mudanças simultâneas em diversas variáveis relevantes do processo o que torna esta operação um dos processos mais complicados observados na prática industrial. Para que se possa propor um procedimento de partida é necessário estudar seu comportamento dinâmico. $\mathrm{O}$ comportamento dinâmico de uma coluna de destilação durante a operação de partida é altamente complexo. O trabalho tem por objetivo o estudo do comportamento dinâmico de um sistema de colunas de destilação termicamente acopladas durante a operação de partida e baseado neste estudo propor um procedimento de partida apropriado para o sistema. O sistema estudado consiste em duas colunas termicamente acopladas no qual uma delas é uma coluna extrativa utilizada para separar a mistura azeotrópica etanol-água tendo como solvente o etilenoglicol. O modelo e o procedimento de partida adotado foram construídos no pacote computacional Aspen Tech. A partir dos resultados foi possível observar o comportamento dinâmico do sistema durante a operação de partida e propor um procedimento adequado.
\end{abstract}

\section{INTRODUÇÃO}

A operação de uma coluna de destilação contínua periodicamente é interrompida para que se possam realizar procedimentos de manutenção. O processo de manutenção tem por objetivo gerar condições operacionais para que equipamentos, instalações e serviços funcionem adequadamente. Após a manutenção, é necessário iniciar a partida da operação da mesma. O procedimento de partida de uma coluna de destilação pode levar algumas horas até dias para atingir o estado estacionário. Quanto maior for o tempo necessário para que a operação da coluna alcance o estado estacionário, maior será a quantidade de produtos fora de especificação e maior será o consumo de energia o que diminui o rendimento do processo e eleva os custos da operação.

Um dos processos mais complicados observados na prática industrial consiste na operação de partida de uma coluna de destilação devido às mudanças simultâneas de diversas variáveis relevantes ao processo (Kruse et al., 1996). 
Durante a operação de partida, o comportamento dinâmico da coluna de destilação apresenta uma tendência que pode ser representada por três estágios característicos. O primeiro estágio, chamado de estágio descontínuo, é caracterizado por seu curto período de tempo e pela natureza descontinua de todas as variáveis. O segundo estágio, o estágio semi-contínuo, é caraterizado pelo comportamento não linear e transiente das variáveis e da aproximação das variáveis hidráulicas de seus valores no estado estacionário. O período de tempo do segundo estágio é maior do que o do primeiro estágio. O terceiro estágio, chamado de estágio contínuo, é caracterizado pelas respostas lineares e transientes de todas as variáveis. No final deste estágio, todas as variáveis atingem seus valores de estado estacionário (Ruiz et al., 1988).Para que se possa desenvolver um procedimento de partida adequado é necessário conhecer e compreender o comportamento dinâmico da coluna de destilação durante a operação de partida.

\section{SISTEMA DE COLUNAS DE DESTILAÇÃO TERMICAMENTE ACOPLADAS}

O sistema de colunas de destilação termicamente acopladas estudado consiste em duas colunas de destilação interligadas por duas correntes, uma corrente na fase vapor (VAP) e uma corrente na fase líquida (LIQ) como representado pela Figura 1.

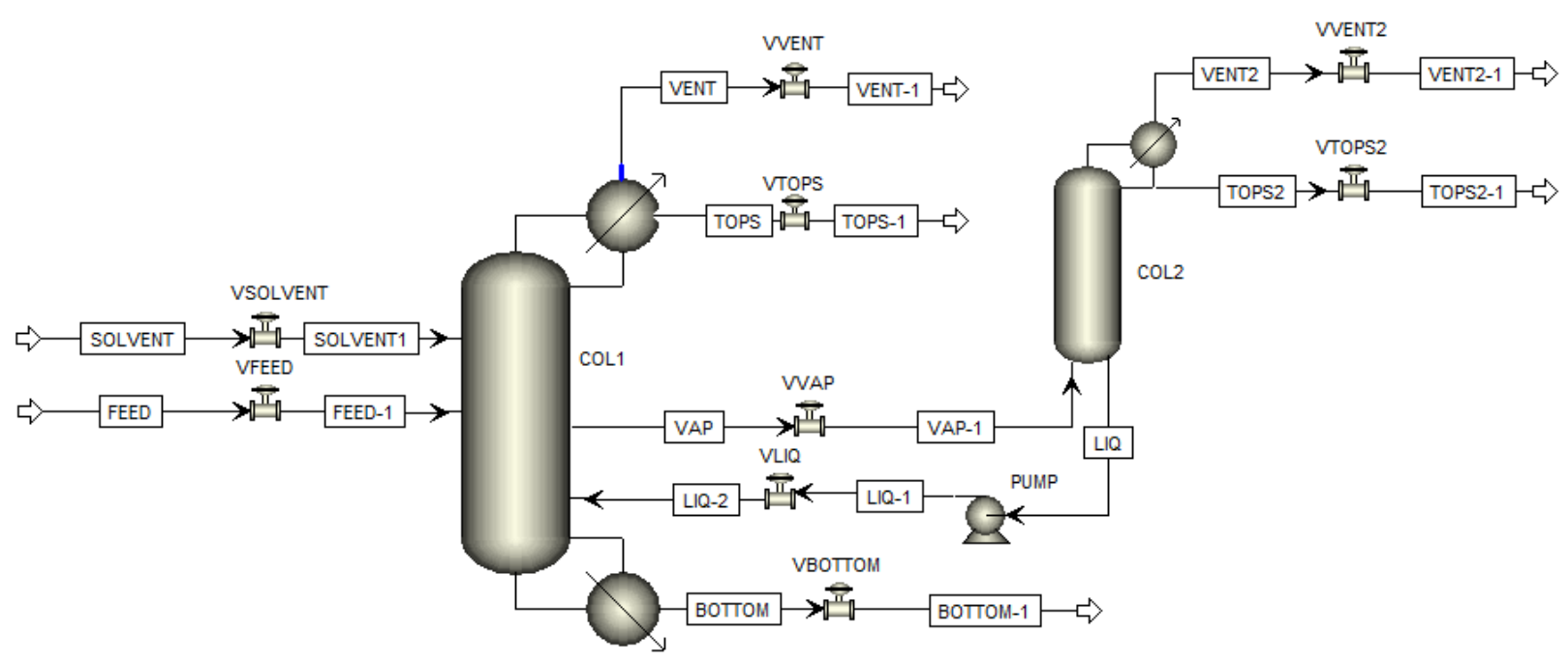

Figura 1 - Fluxograma do sistema de colunas de destilação termicamente acopladas.

Devido à interligação entre as colunas eliminou-se o reboiler da segunda coluna de destilação. A COL1 é uma coluna de destilação extrativa utilizada para separar a mistura azeotrópica etanol-água e tem como solvente o etilenoglicol.

\section{METODOLOGIA}

Inicialmente o modelo do sistema estudado, no estado estacionário, foi construído no Aspen Plus. Depois de alcançada a convergência, este modelo foi transferido para o Aspen Plus Dynamics. 


\section{9 a 22 de outubro de 2014 \\ Florianópolis/SC}

No Aspen Plus Dynamics todos os controladores foram adicionados e os parâmetros ajustados. Executou-se então o Script pré-construído "Empty" que consiste em deixar a coluna no estado vazio e frio, o que corresponde ao seu estado inicial. Na realidade, a coluna não fica vazia, mas cheia de nitrogênio a $20^{\circ} \mathrm{C}$ de temperatura e pressão de 1,01 bar.

Durante a operação de partida o gás inerte, nitrogênio, deve ser purgado do interior da coluna de destilação e, portanto, deve-se ter duas correntes de saída de produto no topo da coluna no modelo construído no Aspen Plus, uma para a saída do produto desejado e outra para a purga do nitrogênio.

Depois de executado o Script "Empty", todos os controladores são colocados em modo manual e os sinais de saída dos controladores ajustados em seus valores mínimos.

Executa-se então o modo de inicialização para que o sistema se ajuste ao estado inicial com todas as vazões iguais a $0 \mathrm{kmol} / \mathrm{hr}$.

Depois de executada a inicialização, adiciona-se um "Task" ao modelo, que consiste no procedimento adotado para a partida da coluna.

Altera-se então o modo de executar para dinâmico e executa-se a simulação.

\section{RESULTADOS}

\subsection{Procedimento de Partida}

No tempo $\mathrm{t}=0$, as válvulas VFEED e VSOLVENT são abertas de modo a iniciar a alimentação da coluna de destilação COL1 com a mistura a ser separada e com o solvente respectivamente. A vazão de entrada da corrente FEED-1 é de $100 \mathrm{kmol} / \mathrm{hr}$ e da corrente SOLVENT-1 é de $90 \mathrm{kmol} / \mathrm{hr}$. Desta forma, o nível da base da coluna começa a aumentar. Quando o nível da base alcança o valor 1,7 $\mathrm{m}$ as válvulas VFEED e VSOLVENT são fechadas e a alimentação da coluna de destilação é interrompida, ou seja, a vazão de entrada da corrente FEED-1 assume o valor de $0 \mathrm{kmol} / \mathrm{hr}$ e a corrente SOLVENT-1 o valor de $0 \mathrm{kmol} / \mathrm{hr}$.Em seguida o modo de operação do controle de pressão da coluna de destilação COL1 é alterado para automático e o set point é especificado como 1 bar. No mesmo instante de tempo, a válvula VVENT é totalmente aberta para purgar o nitrogênio do interior da coluna COL1.

A seguir, a vazão do vapor de aquecimento no reboiler da coluna COL1 sofre um aumento gradual. Primeiramente, a vazão do vapor de aquecimento assume um valor de $2600 \mathrm{~kg} / \mathrm{hr}$. Logo após, assume o valor de $2700 \mathrm{~kg} / \mathrm{hr}$ e em seguida o valor de $3000 \mathrm{~kg} / \mathrm{hr}$. Depois de alguns minutos, a válvula VVENT é fechada e a purga de nitrogênio é interrompida.

Em $\mathrm{t}=1,42 \mathrm{hr}$, o refluxo na coluna COL1 é iniciado com uma vazão de $50,2 \mathrm{kmol} / \mathrm{hr}$. No mesmo instante de tempo, a alimentação da coluna de destilação COL2 é iniciada, pela corrente VAP1, com uma vazão de $5 \mathrm{kmol} / \mathrm{hr}$. Em seguida, o controle de pressão da mesma é colocado em modo de operação automático com o set point de 0,6 bar. A válvula VVENT2 é, então, totalmente aberta para 
que o nitrogênio contido no interior da coluna COL2 possa ser purgado. No tempo $\mathrm{t}=1,57 \mathrm{hr}$, a vazão do vapor de aquecimento é aumentada para $3200 \mathrm{~kg} / \mathrm{hr}$.

Posteriormente, o controle de nível do vaso de refluxo da coluna COL1 é colocado em modo automático com um set point de $2 \mathrm{~m}$. Depois de $12 \mathrm{~min}$, a válvula de purga VVENT2 da coluna COL2 é fechada e a purga de nitrogênio é interrompida. A seguir, a vazão de refluxo é aumentada para 59,2 kmol/hr e a vazão da corrente de alimentação da COL2, VAP-1, aumenta para $25 \mathrm{kmol} / \mathrm{hr}$. No mesmo instante de tempo ocorre o aumento da vazão do vapor de aquecimento no reboiler que assume o valor de $3300 \mathrm{~kg} / \mathrm{hr}$. Após $9 \mathrm{~min}$, o refluxo da COL2 é iniciado à uma vazão de 11,5 $\mathrm{kmol} / \mathrm{hr}$.

Logo após, as válvulas VFEED e VSOLVENT são novamente abertas e alimentação da COL1 reiniciada com as vazões de $100 \mathrm{kmol} / \mathrm{hr}$ e $90 \mathrm{kmol} / \mathrm{hr}$, respectivamente. Assim que o nível na base da coluna COL1 alcança a marca de $1,7 \mathrm{~m}$, as vazões das correntes de alimentação são reduzidas. A vazão da corrente FEED-1 assume o valor de $20 \mathrm{kmol} / \mathrm{hr}$ e a vazão da corrente SOLVENT-1 o valor de $10 \mathrm{kmol} / \mathrm{hr}$. Em seguida, a vazão do vapor de aquecimento sofre um novo aumento e vai para 3600 $\mathrm{kg} / \mathrm{hr}$.

Logo após, a vazão de alimentação da COL2 é aumentada e assume o seu valor final que é 30 $\mathrm{kmol} / \mathrm{hr}$. A seguir, a vazão de refluxo da COL2 é aumentada para 13,5 kmol/hr e a vazão do vapor de aquecimento no reboiler da COL1 sofre um aumento e vai para $3700 \mathrm{~kg} / \mathrm{hr}$.

Posteriormente, o controle de nível da base da coluna COL1 é colocado em modo automático e ocorre o ajuste da vazão de refluxo da coluna COL1 para o valor final, que é de 56,4 kmol/hr. No mesmo instante de tempo, a alimentação da coluna COL1 é ajustada com a vazão de $100 \mathrm{kmol} / \mathrm{hr}$ para a corrente FEED-1 e $90 \mathrm{kmol} / \mathrm{hr}$ para corrente SOLVENT-1. Depois de 1 hora a vazão de refluxo da coluna COL2 é ajustada para 37,8 kmol/hr e a vazão do vapor de aquecimento é aumentada para 4000 $\mathrm{kg} / \mathrm{hr}$ e posteriormente para $5000 \mathrm{~kg} / \mathrm{hr}$.

Espera-se até que a temperatura no estágio 24 alcance o valor de $171,5^{\circ} \mathrm{C}$ para que o controle de temperatura opere em modo automático. Logo após, ajusta-se os valores dos set points dos controles de nível do vaso de refluxo da coluna COL1 para 1,25 m, da base da coluna COL1 para 1,6 m e do vaso de refluxo da coluna COL2 para $0,55 \mathrm{~m}$.

\subsection{Resultados Gráficos}

A Figura 2 mostra o comportamento dinâmico da vazão da corrente FEED-1 e da corrente SOLVENT-1, que correspondem as correntes de alimentação da coluna COL1. 


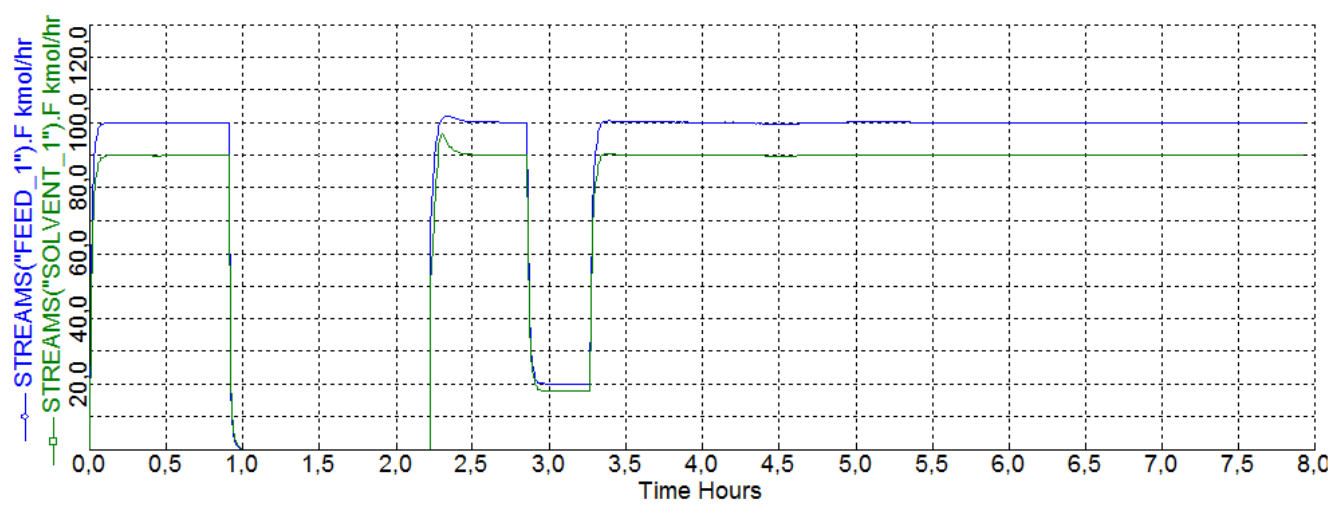

Figura 2 - Vazões de alimentação da coluna de destilação COL1.

Conforme descrito pelo procedimento de partida as vazões de alimentação sofrem variações bruscas até o momento em que a vazões é ajustadas em seus valores finais de $100 \mathrm{kmol} / \mathrm{hr}$ para a corrente FEED-1 e $90 \mathrm{kmol} / \mathrm{hr}$ para a corrente SOLVENT-1.

A Figura 3 mostra o comportamento dinâmico do nível da base da coluna de destilação COL1 durante a operação de partida.

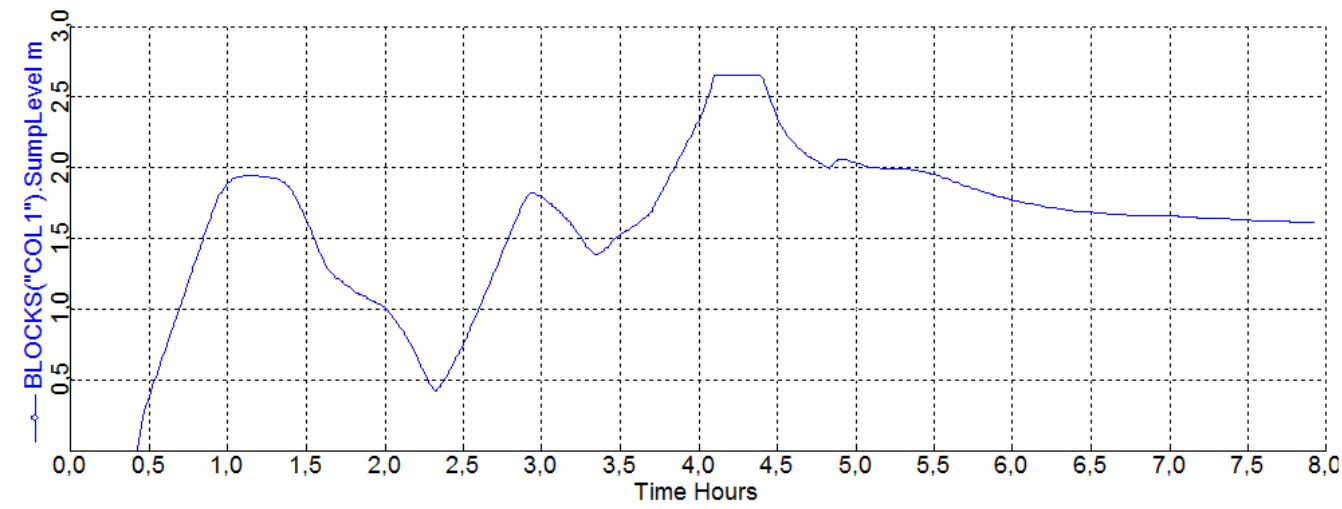

Figura 3 - Nível na base da coluna de destilação COL1.

A Figura 3 mostra que o nível na base da coluna COL1 aumenta quando a alimentação é introduzida na coluna e diminui quando a vazão do vapor de aquecimento no reboiler aumenta fazendo com que o liquido na base se transforme em vapor. A variação brusca do nível ocorre até o momento em que o controle de nível da base é colocado em modo automático.

A Figura 4 apresenta o comportamento dinâmico da vazão do vapor de aquecimento no reboiler da coluna de destilação COL1 durante a operação de partida. 


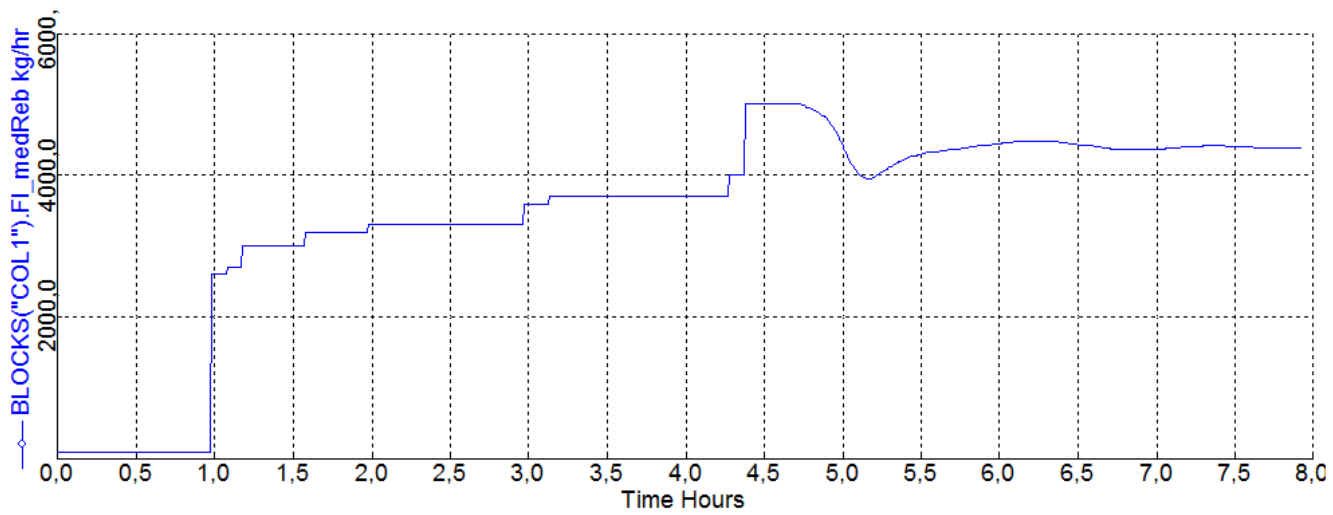

Figura 4 - Vazão do vapor de aquecimento no reboiler da coluna de destilação COL1.

A Figura 4 mostra que conforme descrito pelo procedimento de partida a vazão do vapor de aquecimento no reboiler é aumentada gradualmente no decorrer do tempo até o momento em que o controle de temperatura é colocado em modo automático.

A Figura 5 mostra o comportamento dinâmico do nível do vaso de refluxo da coluna de destilação COL1 durante a operação de partida.

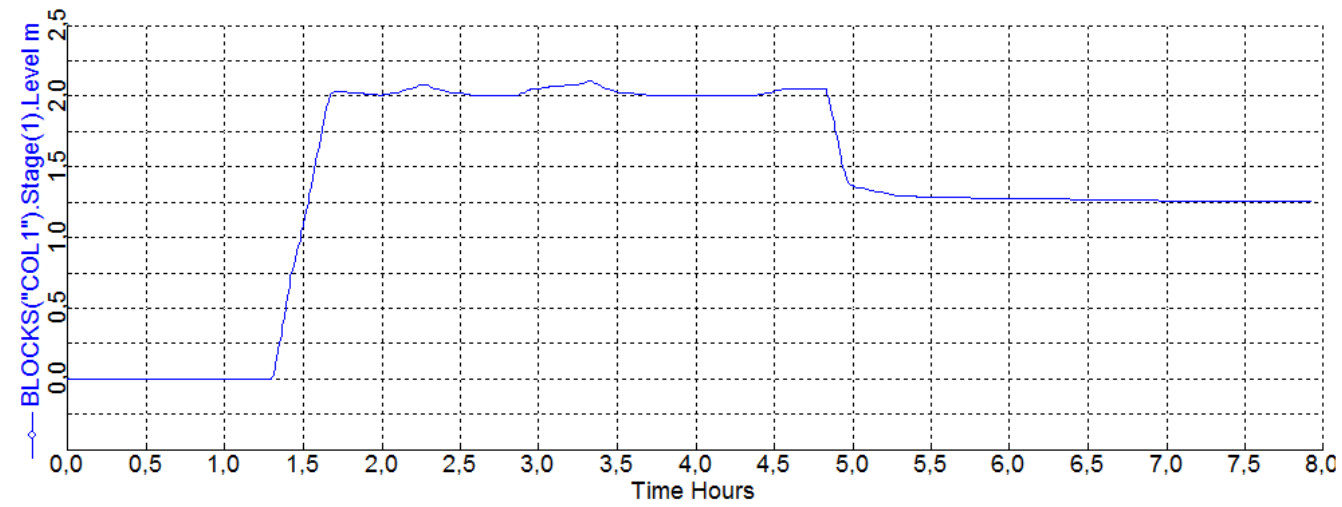

Figura 5 - Nível do vaso de refluxo da coluna de destilação COL1.

A Figura 5 mostra que o nível no vaso de refluxo no decorrer do procedimento de partida não sofre grandes variações permanecendo próximo a $2 \mathrm{~m}$ e só é modificado no final da operação de partida.

A Figura 6 mostra o comportamento dinâmico do nível do vaso de refluxo da coluna de destilação COL2 durante a operação de partida. 


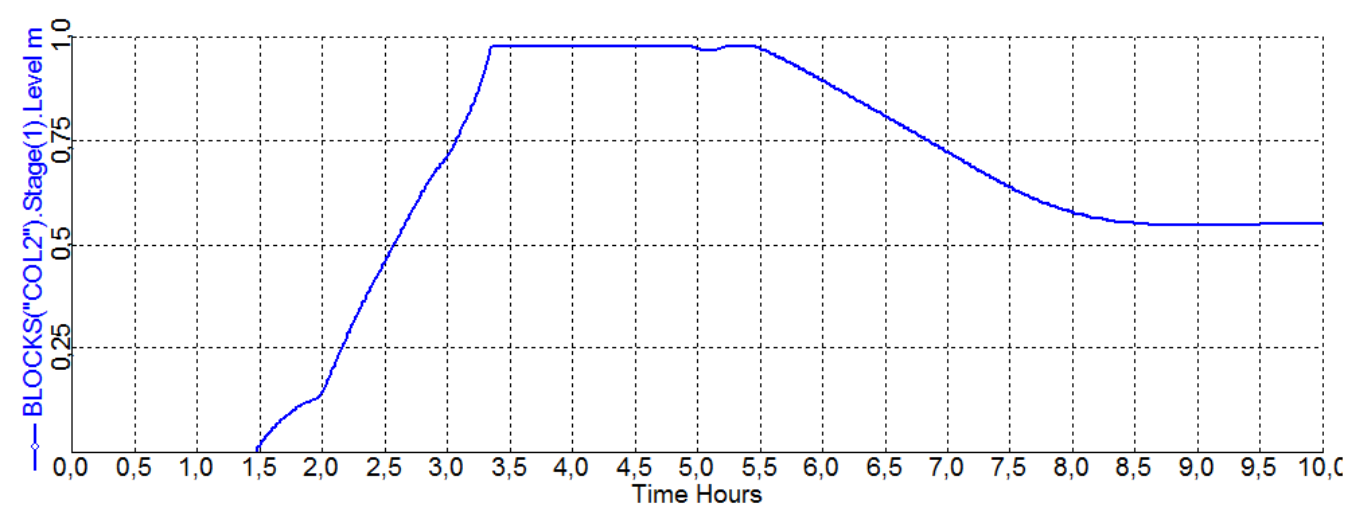

Figura 6 - Nível do vaso de refluxo da coluna de destilação COL2.

A Figura 7 mostra o comportamento dinâmico da composição da corrente de produto TOPS-1 da coluna de destilação COL1 durante a operação de partida.

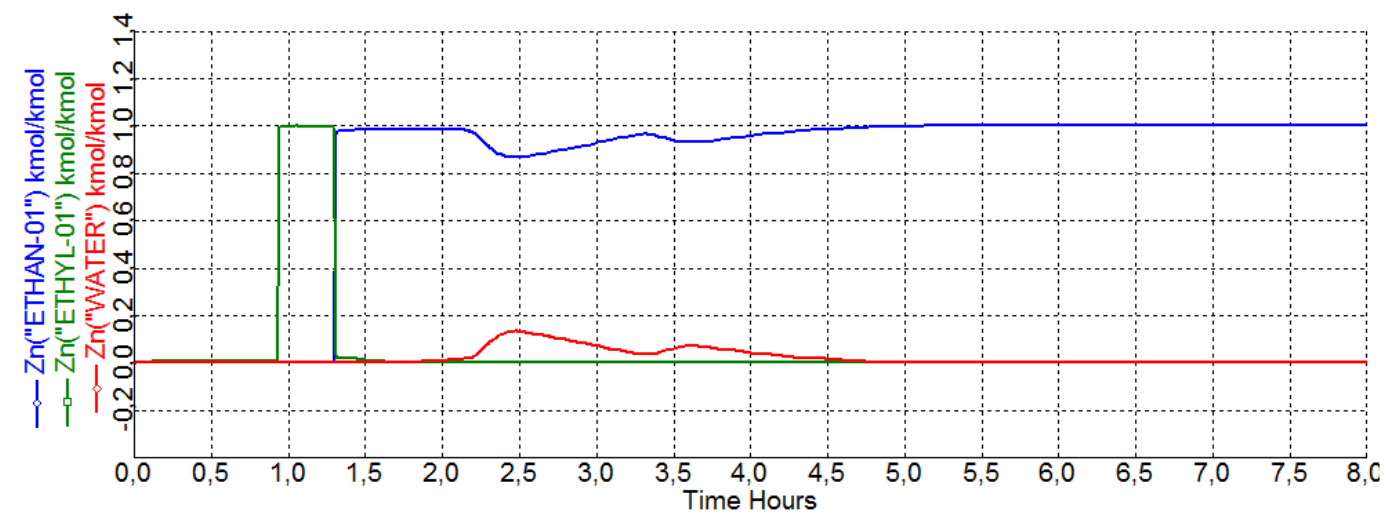

Figura 7 - Composição da corrente TOPS-1 da coluna de destilação COL1.

Como o etilenoglicol é alimentado no prato 4, assim que ele entra na coluna ocorre uma vaporização parcial fazendo com que a composição do solvente no topo aumente. No decorrer da partida ocorre a separação do azeótropo etanol-água e a composição do etanol no topo aumenta e a composição do etilenoglicol diminui. Além disso, ocorrem variações nas composições do etanol e da água devido às variações na vazão de refluxo.

A Figura 8 mostra o comportamento dinâmico da composição da corrente de base BOTTOM-1 da coluna de destilação COL1 durante a operação de partida. 


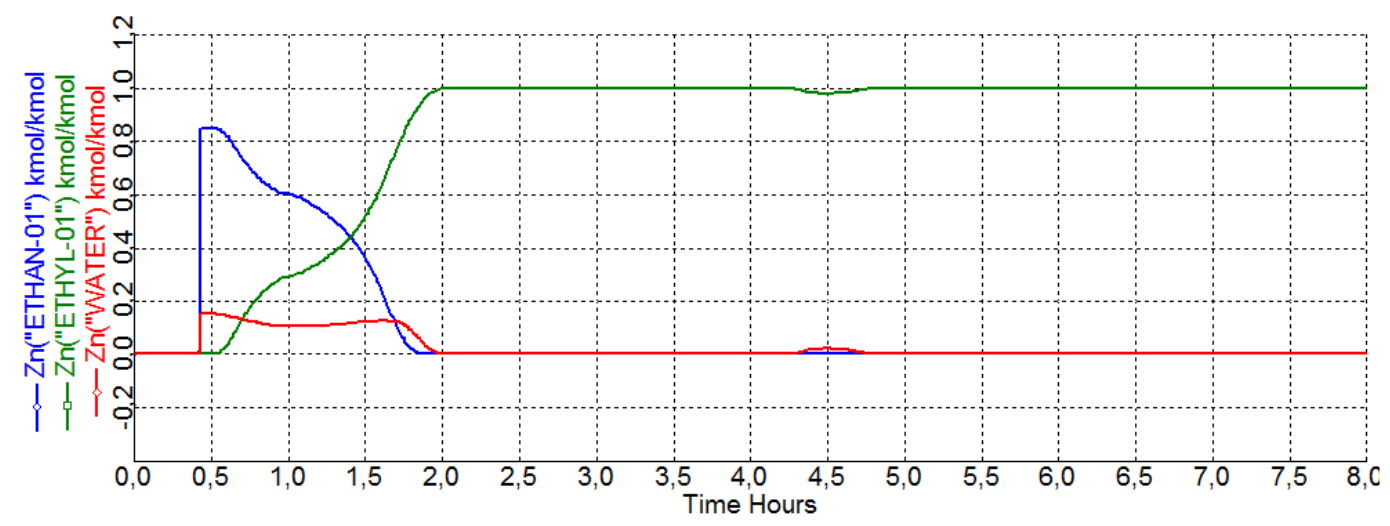

Figura 8 - Composição da corrente BOTTOM-1 da coluna de destilação COL1.

A Figura 9 mostra o comportamento dinâmico da composição da corrente de produto TOPS2-1 da coluna de destilação COL2 durante a operação de partida.

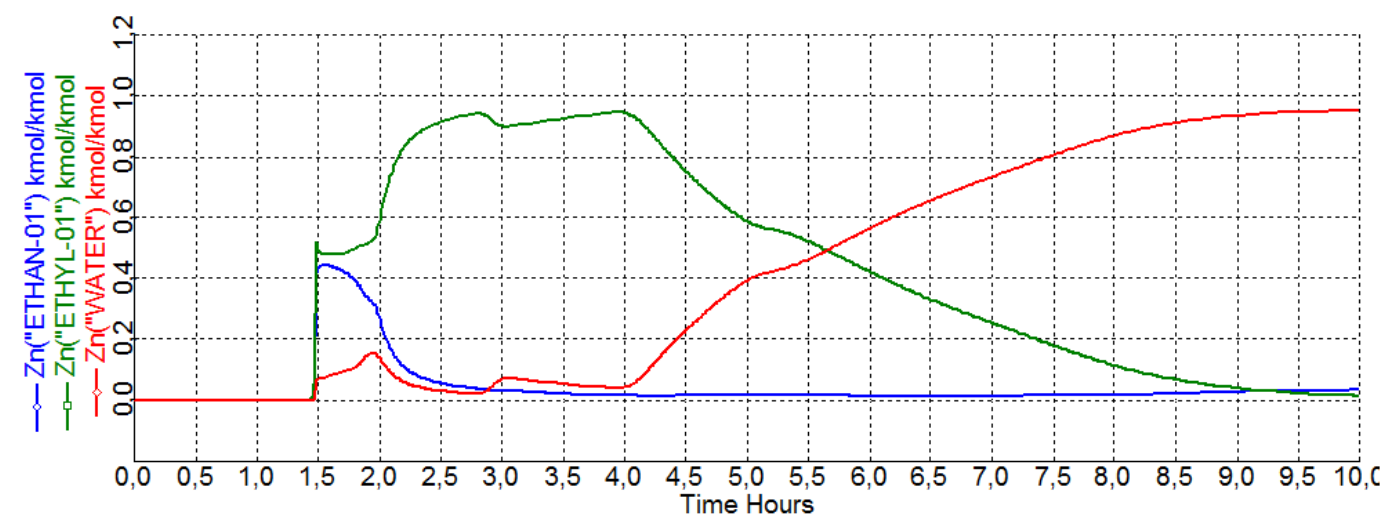

Figura 9 - Composição da corrente TOPS2-1 da coluna de destilação COL1.

Na Figura 9 observa-se que a medida que a vazão de refluxo da coluna COL2 aumenta a composição da água no topo da coluna aumenta e a composição do etilenoglicol diminui.

\section{CONCLUSÕES}

A partir do modelo desenvolvido no Aspen Tech foi possível analisar o comportamento dinâmico da operação de partida do sistema de colunas de destilação termicamente acopladas e propor um procedimento adequado de forma satisfatória.

\section{REFERÊNCIAS}

KRUSE, C.; FIEG, G.; WOZNY, G. A new time-optimal strategy for column startup and product changeover. J. Proc. Cont., v. 6, p. 187-193, 1996.

RUIZ, C. A.; CAMERON, I. T.; GANI, R. A generalized dynamic model for distillation column - III. Study of startup operations. Comput. Chem. Eng., v. 12, p. 1-14, 1988. 